\title{
Indicators for the performance assessment of road bridges through monitoring
}

\author{
Peter Tanner ${ }^{1}$, Miguel Prieto ${ }^{2}$ \\ DOI: $\underline{\text { https://doi.org/10.5592/CO/BSHM2017.5.1 }}$ \\ ${ }^{1}$ Eduardo Torroja Institute for Construction Science - Spanish National Research Council, IETcc-CSIC \\ (Serrano Galvache 4, Madrid, 28033, Spain) \\ ${ }^{2}$ Eduardo Torroja Institute for Construction Science - Spanish National Research Council, IETcc-CSIC \\ (Serrano Galvache 4, Madrid, 28033, Spain) \\ E-mails: ${ }^{1}$ tannerp@ietcc.csic.es; ${ }^{2}$ mprietor@ietcc.csic.es.
}

\begin{abstract}
Different causes may lead to the non-compliance of a particular requirement related with an existing infrastructure. Often, they may be traced back to deviations from expected actions or resistances. The quantification of parameters related with such influences may provide evidence about the degree of compliance of a given structure with a particular serviceability or safety requirement. Such parameters may therefore be called indicators and associated threshold values can be established on a risk basis, as well as admissible average frequencies for outcrossing.

Indicators can be monitored comparing the measured values continuously to the previously established threshold values. Alarm systems may be installed which are activated in case of outcrossing. Safety measures can therefore be adopted depending on the consequences of the observed non-compliance. Based on such an approach and by using modern information technology, inspections of large infrastructures may be automated and optimised.

A series of indicators for use in road bridge inspection are proposed hereunder, together with their respective threshold values and allowable mean frequency of outcrossing. The paper also includes a practical application in which these criteria are applied to a road bridge with an unknown reliability level.
\end{abstract}

Keywords: Uncertainties, assessment, monitoring, road bridges, indicators, threshold values

\section{Introduction}

Structural systems must be engineered, built, operated and maintained to ensure economically sound use throughout their service life, while meeting predefined serviceability and structural safety requirements. Such requirements must be fulfilled with an acceptable level of reliability that depends on a number of parameters, including the reference period considered and possible consequence of non-compliance or failure.

Different influences and circumstances may underlie the non-compliance of a requirement:

- deviations from the assumed values for actions or the effects of environmental influences;

- deviations from values established for other influences, such as construction execution inaccuracies;

- geotechnical actions;

- chemical, physical and biological actions;

- actions or influences not considered;

- dynamic effects such as resonance;

- deviations from the structural or soil strength values assumed;

- strength loss due to decay mechanisms such as corrosion, embrittlement or fatigue;

- structural overloads or strength losses induced by accidental actions. 
A structure's compliance with predefined requirements can be determined by quantifying parameters associated with these influences or circumstances, which are consequently called indicators and, generally speaking, refer to system geometry, materials, actions or structural behaviour. The parameters chosen for effective inspection and monitoring should be the ones whose variation has the greatest effect on the reliability of the system studied. Hence, parameter selection depends on the type of technical system involved, its purpose and operation, exposure conditions, constituent materials and the data acquisition resources available.

When inspection planning is based on the adoption of suitable indicators, its scope can be adapted to the condition of the system elements, which can be prioritised in keeping with their significance and the decay mechanisms observed. From this perspective, the use of indicators to define inspection strategy can be interpreted as a risk reduction measure.

The objective of this paper is to define the grounds for the early detection of possible damage or anomalies with a view to adopting risk mitigation measures before an undesired event such as structural collapse occurs. Inspection scope and intensity should be determined on the basis of the characteristics and significance of the structure inspected, as well as of the allowable risk.

\section{Scope and assumptions}

The present contribution summarizes the principles for the definition of the inspection and maintenance activities for bridges throughout their service life, to be established in detail for each individual case within the framework of a specific bridge project (Tanner and Prieto (2013a, 2013b)). These principles are applicable to all bridges, at all construction and use stages. They are also applicable to temporary load bearing structures, as well as for the assessment of existing bridges, related to refurbishments, changes of use, repair or strengthening.

The specific rules established refer to road bridges, regardless of their constituent materials. The principles defined in this paper should be used in conjunction with a consistent set of standards and recommendations such as the Spanish codes for actions on road bridges, IAP-11 (2011), and the design of steel-, EAE-11 (2011), concrete-, EHE-08 (2008), and composite bridges, RPX-95 (1996). Alternatively, they may also be used together with the respective Eurocode rules.

\section{Inspection}

Depending on the context in which inspection is conducted and the objectives and resources used, a distinction can be drawn among the following:

- observation;

- $\quad$ periodic inspection;

- $\quad$ control measurements;

- monitoring.

Observation is understood to mean the perception, at pre-defined intervals, of the condition of a structure and its performance. Observations may also be conducted on the occasion of the performance of other tasks, such as maintenance. The qualitative determination and appraisal of the condition of a structure during inspections conducted at pre-defined intervals in accordance with given priorities constitute what is known as periodic inspection. Control measurements may be taken to quantify certain parameters representative of the structure or its performance. Lastly, monitoring consists of determining the condition of a structure through frequent or continuous recording of certain parameters representative of the system or its performance and the comparison of the values recorded to the respective thresholds.

The thresholds to which the data acquired with appropriate techniques and instruments are to be compared must be established depending on the level of reliability associated with each requirement. Information on how to determine thresholds is furnished in section 4 below. The measures that should be adopted when the acquired values for a given indicator exceed one or several of the associated thresholds are also discussed. 

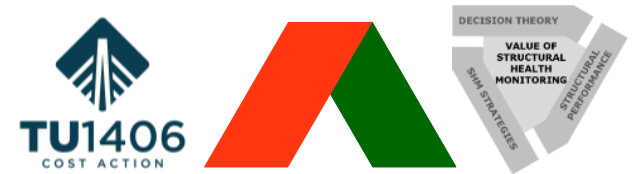

The data acquisition instruments used to monitor bridges must be able to record their static (lasting geometric changes) and dynamic (instantaneous geometric changes) behaviour. Moreover, for the data recorded to be useful in the long run, the instruments used must be able, at any given time, to establish the ratio between the existing and initial values. Thanks to recent progress in data acquisition, transmission and processing technologies, most parameters relevant to structural performance, particularly geometric change, can be continuously monitored. The effectiveness of inspection and maintenance measures can be considerably enhanced by systematically and suitably installing modern measuring systems on large infrastructures. One example of such a system can be found in the optical fibre sensors, which deliver resolution on the order of thousandths of a millimetre and feature stability over time.

Data acquisition on the established indicators forms part of programmed bridge inspections and should not be confounded with data collection for structural assessment. The need to assess structural reliability may be the outcome of inspection findings, where, for instance, the indicators quantified exceed the respective thresholds (section 4).

\section{Assessment of threshold values associated with serviceability and structural safety}

\subsection{General}

As in the determination of action effects in serviceability or structural safety calculations, the threshold values for the parameters quantified during road bridge inspections, known as reliability indicators, are normally established by structural analysis. Such analysis are based on the principles laid down in a consistent set of standards and recommendations such as the aforementioned national codes IAP-11 (2011), EHE-08 (2008), EAE-11 (2011) and RPX-95 (1996) or the respective Eurocodes. Where existing bridges are involved, the updated values for the relevant variables should be used for the analysis, and where no updated information is available, the nominal values can often be applied.

Structural analysis should deploy parameters able to predict a bearing structure's performance sufficiently accurately for the control situations studied. The methods used to these ends must be backed by substantiated theory. The analytical model must integrate actions and other influences, geometric data and the properties of the materials constituting the structure and the terrain.

\subsection{Requirements}

\subsubsection{Overview}

Table 1 lists the requirements for road bridges, irrespective of the constituent materials, in increasing order of the consequences of possible non-compliance. Indicators are established and the respective threshold values are also given, along with the allowable mean frequency of transgression of the associated requirements. Taking a 1 year reference period as a basis, both the threshold values and the admissible frequencies of transgression are established in such a way to represent an acceptable level of structural performance per unit of time, equivalent to that associated with permanent structures according to the applied code requirements (see also item 4.2.3). The measures to be adopted in the event of non-compliance of any of these requirements are mentioned in item 4.3 below.

The choice of indicators is conditioned by the availability of suitable data acquisition or measuring facilities. In the aforementioned study (Tanner and Prieto (2013a, 2013b)), monitoring was usually, but not exclusively, conducted with fibre optic sensors. As a general rule, when threshold values are determined on the grounds of structural analysis, monitoring can only detect the effects of actions applied after a new or existing bridge is instrumented. 


\section{INDICATORS FOR THE PERFORMANCE ASSESSMENT OF ROAD BRIDGES THROUGH MONITORING}

Table 1. Requirements, indicators and thresholds values associated with road bridge serviceability (SLS) and structural safety (ULS).

\begin{tabular}{|c|c|c|c|c|c|c|}
\hline & \multirow[b]{2}{*}{ Demand } & \multirow[b]{2}{*}{ Consequences } & \multirow[b]{2}{*}{ Requirement } & \multirow[b]{2}{*}{ Indicator } & \multicolumn{2}{|c|}{ Threshold } \\
\hline & & & & & $\begin{array}{c}\text { Value } \\
E_{\mathrm{ser}, \text { lim }} ; C_{\mathrm{ser}, \mathrm{lim}} ; E_{\mathrm{d}, \mathrm{lim}} \\
\end{array}$ & $\begin{array}{c}\text { Mean frequency } \\
\omega_{\text {ser; }} \omega_{\mathrm{d} ;} \\
\end{array}$ \\
\hline \multirow{8}{*}{$\frac{n}{n}$} & Appearance & Reversible & Deformations & Deflection & $\mathrm{L} / 700^{1)}$ & $50 \%$ of time \\
\hline & Appearance & Reversible & Deformations & Strain & $E_{\text {ser, }, \lim , 2}$ & $50 \%$ of time \\
\hline & Comfort & Reversible & Deformations & Deflection & $\mathrm{L} / 1000^{2)}$ & Weekly \\
\hline & Comfort & Reversible & Deformations & Strain & $E_{\text {ser, lim }, 1}$ & Weekly \\
\hline & \multirow{4}{*}{$\begin{array}{l}\text { Comfort } \\
-\quad \text { Maximum } \\
-\quad \text { Medium } \\
-\quad \text { Minimum }\end{array}$} & \multirow{4}{*}{ Reversible } & \multirow{4}{*}{ Vibrations } & \multirow{4}{*}{ Acceleration } & $a_{v}{ }^{3)}$ & \\
\hline & & & & & 0.5 & - \\
\hline & & & & & 1.0 & - \\
\hline & & & & & 2.5 & - \\
\hline \multirow{4}{*}{ s } & $\begin{array}{l}\text { Structural } \\
\text { reliability }\end{array}$ & Reversible & $\begin{array}{l}\text { Safety of structure } \\
\text { and facilities }\end{array}$ & Traffic loads ${ }^{4)}$ & $E_{d, \lim , 1}$ & Weekly \\
\hline & $\begin{array}{l}\text { Structural } \\
\text { reliability }\end{array}$ & Reversible & $\begin{array}{l}\text { Safety of structure } \\
\text { and facilities }\end{array}$ & Strain & $E_{d, \lim , 1}$ & Weekly \\
\hline & $\begin{array}{l}\text { Structural } \\
\text { reliability }\end{array}$ & Irreversible & Safety of people & Traffic loads $^{4)}$ & $E_{d, \lim , 0}$ & Yearly \\
\hline & $\begin{array}{l}\text { Structural } \\
\text { reliability }\end{array}$ & Irreversible & Safety of people & Strain & $E_{d, \lim , 0}$ & Yearly \\
\hline \multicolumn{7}{|c|}{$\begin{array}{l}\text { 1) Deflection after subtracting possible precamber, bearing in mind long-term effects due to creep, shrinkage and } \\
\text { relaxation } \\
\text { 2) Deflection due to traffic loads; higher values, up to L/500, acceptable for existing bridges on low traffic capacity } \\
\text { roads }\end{array}$} \\
\hline
\end{tabular}

\subsubsection{Threshold values associated with serviceability}

A bridge is regarded as serviceably sound if the values of the parameters measured during inspection are non-compliant with condition (1) less often than the allowable frequency, $\omega_{\text {ser, }}$ associated with the respective threshold value:

$E_{\text {mon }} \leq E_{\text {ser,lim }}$

$E_{\mathrm{mon}} \quad$ measured value of a given indicator

$E_{\text {ser,lim }}$ threshold value for the same indicator, associated with serviceability

For some indicators associated with serviceability requirements such as deflection due to traffic loads or acceleration induced by dynamic actions in bridges with pedestrian access, standard IAP-11 (2011) lists indicative thresholds for in-service structural performance parameters. Given that these values may be adopted as serviceability thresholds $\left(C_{\text {ser,lim }}\right)$ with no need to conduct structural analysis, bridge 

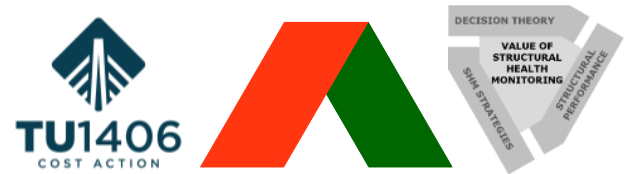

performance may be regarded as suitable where the values measured for the respective parameter are non-compliant with condition (2) less often than the allowable frequency, $\omega_{\text {ser }}$ :

$$
E_{\text {mon }} \leq C_{\text {ser,lim }}
$$

The threshold value for action effects associated with the relevant control situations that should not be exceeded more than once a week can be determined from the following expression (symbols defined as in standard IAP-11 (2011)):

$$
E_{\text {ser,lim, }, 1}=E\left(\sum_{j \geq 1} G_{k, j} "+" P "+" \psi_{1,1} \cdot Q_{k, 1} "+" \sum_{i>1} \psi_{2, i} \cdot Q_{k, i}\right)
$$

The threshold value for action effects associated with the relevant control situations that should not be exceeded more than $50 \%$ of the time can be determined from the following expression (symbols defined as in standard IAP-11 (2011)):

$$
E_{\text {ser,lim, }, 2}=E\left(\sum_{j \geq 1} G_{k, j} "+" P^{\prime \prime}+" \sum_{i \geq 1} \psi_{2, i} \cdot Q_{k, i}\right)
$$

In the absence of more detailed analysis, the values of the factors for combination values of actions $\psi_{1}$ and $\psi_{2}$ can be taken from standard IAP-11 (2011) or the respective Eurocode EN 1990:2002/A1:2005 (2005).

\subsubsection{Threshold values associated with structural safety}

A bridge is regarded as structurally safe if the values of the parameters measured during inspection are non-compliant with condition (5) less often than the allowable frequency, $\omega_{d}$, associated with the respective threshold value:

$E_{\text {mon }} \leq E_{d, \lim }$

$E_{m o n} \quad$ measured value of a given indicator

$E_{\text {d,lim }}$ threshold value for the same indicator, associated with structural safety

The threshold value for action effects associated with the relevant control situations that should not be exceeded more than once a year can be determined from the following expression, in which the symbols are defined as in standard IAP-11 (2011):

$$
E_{d, \mathrm{lim}, 0}=E\left(\sum_{j \geq 1} \gamma_{G, j} \cdot G_{k, j} "+" \gamma_{P} \cdot P "+" \gamma_{Q, 1} \cdot \psi_{0,1} \cdot Q_{k, 1} "+" \sum_{i>1} \gamma_{Q, i} \cdot \psi_{1, i} \cdot Q_{k, i}\right)
$$

The threshold value for action effects associated with the relevant control situations that should not be exceeded more than once a week can be determined from the following expression (symbols defined as in standard IAP-11 (2011)):

$$
E_{d, \mathrm{lim}, 1}=E\left(\sum_{j \geq 1} \gamma_{G, j} \cdot G_{k, j} "+" \gamma_{P} \cdot P "+" \gamma_{Q, 1} \cdot \psi_{1,1} \cdot Q_{k, 1} "+" \sum_{i>1} \gamma_{Q, i} \cdot \psi_{2, i} \cdot Q_{k, i}\right)
$$

In cases where non-compliance of safety criteria may involve risks to persons, the partial factors for actions to be used for the establishment of the threshold values should be based on life-safety riskrelated acceptance criteria associated with current best practice. Using a life safety risk metric proposed in Faber et al. (2015), which relates risk exposure due to different activities and applied technologies, the general approach for the definition of the time-dependent safety requirements is to maintain the acceptable life safety risk per time unit during the reference period considered for the use of a structure at the same level as for structures under permanent use conditions. 


\section{INDICATORS FOR THE PERFORMANCE ASSESSMENT OF ROAD BRIDGES THROUGH MONITORING}

In the absence of more detailed analysis, and assuming that the investigated bridge is correctly designed to consistent code specifications, the values of the partial factors for actions $\gamma_{G}, \gamma_{P}$ and $\gamma_{Q}$ as well as of the factors for combination values of actions $\psi_{0}, \psi_{1}$ and $\psi_{2}$ can be taken from standard IAP11 (2011) or the respective Eurocodes, EN 1990 (2002), EN 1990:2002/A1:2005 (2005).

\subsection{Planning and adoption of measures}

If any of the requirements set out in Table 1 is not met, suitable corrective measures must be taken to mitigate the possibility of personal harm or environmental or economic damage. Such measures must be carefully planned, which necessarily entails an analysis of both, the cause or causes of the noncompliance observed and of structural reliability. In certain cases, such as where fluctuations in the mean or extreme values of a given indicator are observed to accelerate or follow a trend, it may be advisable to analyse the values recorded even where no non-conformity has been formally established.

\section{Bridge monitoring application}

\subsection{Introduction}

The proposed methodology was applied to a bridge of unknown reliability, located in the province of Seville, Spain. The bridge chosen runs in the east-west direction and crosses the River Guadalquivir. This five-span $(40+68+100+68+40 \mathrm{~m}), 316 \mathrm{~m}$ long composite bridge features a variable depth (Figure 1a). Its approach viaduct consists of a six-span $(4 \times 27+30+22 \mathrm{~m}), 160 \mathrm{~m}$ long continuous, post-tensioned slab which lies outside of the scope of the study.

The bridge deck is $30.1 \mathrm{~m}$ wide. Its triple-cell composite box girder varies in plan and elevation view dimensions. The width of the bottom flange of the steel box ranges from 9.2 to $15.4 \mathrm{~m}$ while its depth gradually rises from 2.25 at the mid-point in the $100 \mathrm{~m}$ span to $4.55 \mathrm{~m}$ over piers P-2 and P-3. It is fitted with longitudinal and transverse stiffeners, as well as diaphragms placed at $4 \mathrm{~m}$ intervals, in addition to the support diaphragms. The concrete slab is $0.22 \mathrm{~m}$ thick with passive (two layers $\phi 20$ at 0.15 ) and active (48 tendons $7 \phi 0.6$ " in the sections on piers P-1 and P-4 and 96 tendons in the ones on piers P-2 and P-3) reinforcements. It has pile foundations, confined (Pot-type) neoprene bearings at abutment 1 (E-1), P-1, P-2, P-3 and P-5 and hooped neoprene bearings at P-4.

a)

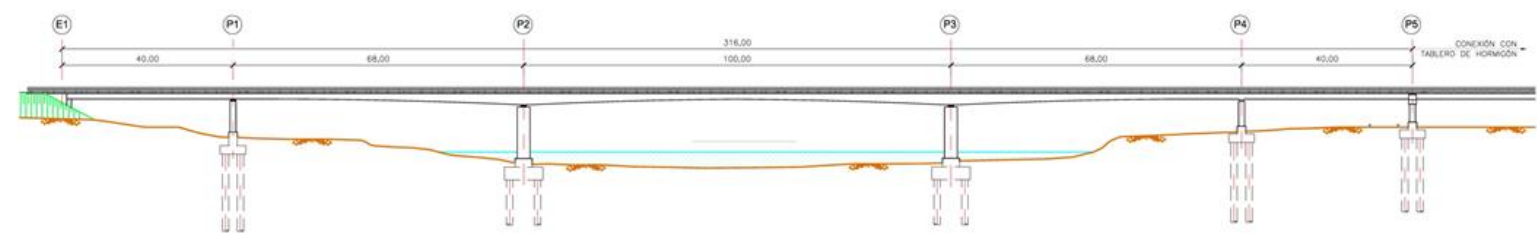

b)

c)

d)

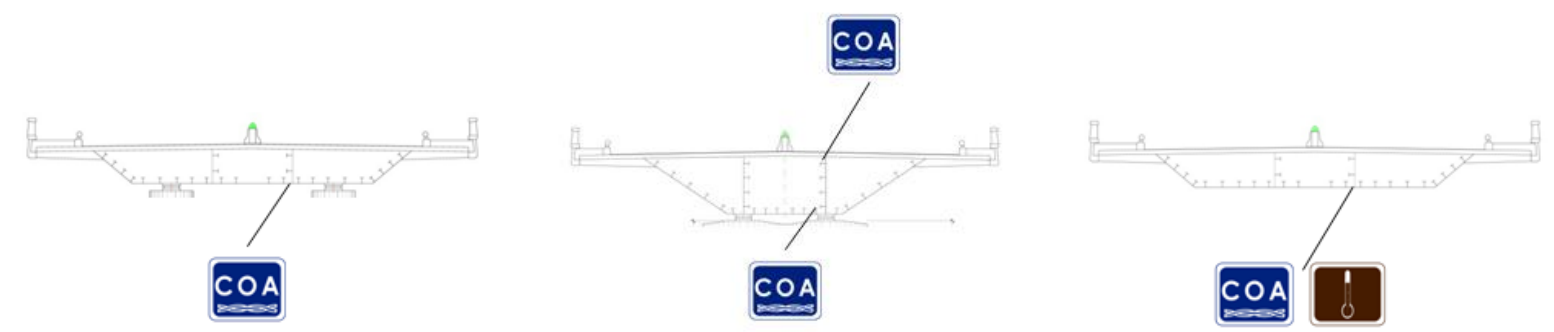

Fig. 1. Investigated bridge and location of sensors: a) longitudinal section; b) cross section P-1; c) cross section P-2; d) main span cross section 

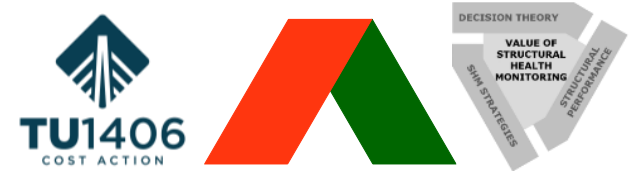

\subsection{Monitoring system and analytical model}

The indicator used to monitor the bridge was unit strain. Given bridge symmetry and taking into account the resolution of the data acquisition system used, only one half of the structure was instrumented. The system chosen to monitor unit strain consisted of $2 \mathrm{~m}$ long fibre optic sensors inside steel protective sheaths. These sensors were able to log changes in shape and position to a precision of $0.01 \mathrm{~mm}$ for static readings ( 1 every 10 minutes) and $0.001 \mathrm{~mm}$ for dynamic readings (50 per second). The static values logged were the mean of the readings taken in each 10-minute interval.

Three sections were chosen to measure unit strain in the longitudinal direction of the bridge (Figure 1): the two sections with maximum hogging moments over piers P-1 and P-2, which, moreover, concurred with the sections actively reinforced, and the one with maximum sagging moments (mid-point in the $100 \mathrm{~m}$ span). Two optical sensors were positioned in the steel cross-section over pier P-2, one on the lower flange and the other on the upper flange (Figure 1c). Pier P-1 (Figure 1b) and the centre of the $100 \mathrm{~m}$ span (Figure 1d) were instrumented only on the upper face of the lower flange. The temperature inside the box was recorded in the central cross-section of the main span.

Structural calculations were performed with Civilcad2000 (2000) software. Since the structure rests on deep foundations consisting of piles, analysis of the composite deck alone, i.e., separate from the substructure, was deemed sufficient. The construction stages addressed in the analysis of the composite structure included positioning of the steel structure, concrete casting in 5 stages and subsequent cable prestressing in the upper slab, concrete casting on five non-prestressed stretches of the upper slab, concrete casting of the lower slab and prestressing of external post-tensioning. Taking into account that the bridge has been in service for several years when the aforementioned data acquisition system has been installed, the actions studied to assess the structure were self-weight, traffic loads, pedestrians, wind, as well as imposed deformations due to settlement and temperature.

From the stress values obtained in the extreme fibres of the sections studied with the numerical model for the relevant combinations of actions, unit strains were found assuming elastic structural behaviour. As the structure's self-weight, dead loads, prestressing forces and imposed deformations were applied before the installation of the sensors, the established threshold values did not include the respective unit strains (item 4.2.1).

\subsection{Comparisons of the findings to threshold values}

Data acquisition was available only for 2 months due to budget restrictions. Figure 2 compares the data logged during that time by the sensor positioned on the upper face of the bottom flange in the cross-section over pier P-1 to the respective thresholds. The convention used here was to show compression as negative and tensile strains as positive. Serviceability threshold $E_{\text {ser,lim, } 1}$ was not exceeded and $E_{\text {ser,lim,2 }}$ was exceeded less than $50 \%$ of the time. The readings were also well within structural safety thresholds $\mathrm{E}_{d, \mathrm{lim}, 1}$ and $\mathrm{E}_{d, \mathrm{lim}, 0}$, which is consistent with the fact that the structure was designed to bear traffic loads characteristic of expressway bridges, whereas its traffic is primarily urban.

\section{Final remarks}

This paper defines a procedure for monitoring road bridges with a view to early detection of possible damage or anomalies to be able to adopt suitable measures before an undesired event such as structural collapse occurs. It contains proposals for indicators or quantifiable parameters that can provide information on the degree of compliance with serviceability and structural safety requirements. It also discusses recommended threshold values and the mean frequency with which they may be exceeded before risk mitigation measures are required.

The procedure proposed was applied to a road bridge with an unknown reliability level. The monitoring was in place for a relatively short time, but the findings suggest that long-term analysis of the data so acquired could be an improvement for the effectiveness of road bridge inspection and maintenance. 


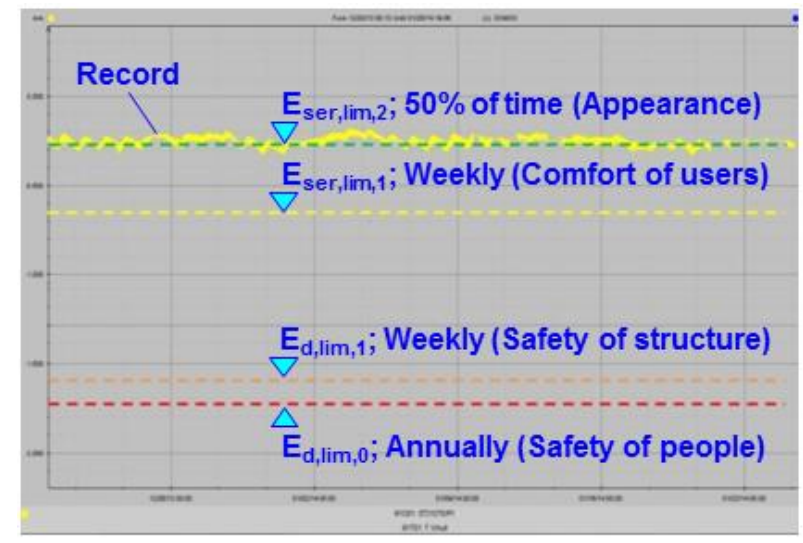

Fig. 2. Results from monitoring with fiber optic sensors on bottom flange of the cross-section over pier P-1 and comparison with threshold values associated with serviceability and structural safety requirements

\section{Acknowledgements}

This study was conducted under the SEGUSTRUC project entitled "Investigación y desarrollo en el control de la seguridad estructural con nuevos sensores basados en tecnología de fibra óptica", funded by the Centre for Industrial Technological Development (Spanish initials, CDTI) and awarded to Euroconsult S.A. The collaboration of Álvaro Navareño Rojo, technical adviser to the Deputy Directorate for Conservation of the Spanish Ministry of Internal Development's Directorate General of Roads, and Emilio Asensio García, Chief Officer of Conservation and Operations of the Western Andalusian Department of Roads, enabling the monitoring of the bridges investigated under the SEGUSTRUC project is gratefully acknowledged.

\section{References}

Civilcad2000 (2000), CivilCAD Consultores S.L.

EAE (2011). Structural steel standard (Written in Spanish). Madrid. Ministerio de Fomento.

EHE-08 (2008). Structural concrete standard (Written in Spanish). Madrid, Ministerio de Fomento.

EN 1990 (2002). Eurocode. Basis of structural design. Brussels. CEN.

EN 1990:2002/A1:2005 (2005). Basis of structural design. Annex A2: Application for Bridges. Brussels. CEN.

Faber et al. (2015). On the regulation of Life Safety Risk, 12th International Conference on Applications of Statistics and Probability in Civil Engineering, ICASP 12, Vancouver, Canada, July 12-15, 2015.

IAP-11 (2011). Actions for the design of road bridges (Written in Spanish). Madrid. Ministerio de Fomento.

RPX-95 (1996). Design recommendations for composite road bridges (Written in Spanish). Madrid. Ministerio de Fomento.

Tanner and Prieto (2013a). Theoretical basis for the inspection and maintenance project of road bridges. Technical Report $\mathrm{n}^{\mathrm{o}}$ 19.884-I (Written in Spanish). Madrid. Instituto de Ciencias de la Construcción Eduardo Torroja. IETcc-CSIC.

Tanner and Prieto (2013b). Recommendations for the inspection and maintenance project of road bridges. Technical Report no 19.884-II (Written in Spanish). Madrid. Instituto de Ciencias de la Construcción Eduardo Torroja. IETcc-CSIC. 